\title{
Diferenças entre o Desenvolvimento da Morfologia Derivacional e Flexional no Português Brasileiro no Ensino Fundamental
}

\author{
Differences in the Development of Derivational and Flexional Morphology \\ in Brazilian Portuguese
}

\author{
Márcia Maria Peruzzi Elia da Mota*, ${ }^{a, b}$, Silvia Brilhante Guimarães ${ }^{c}$, Carolina Conti $^{c}$, \\ Tiago Linhares ${ }^{c}$, Leonice Bárbara Rezende ${ }^{c}$, Silvia Amorin ${ }^{c}$, Lívia Coelho $^{c}$, \\ Auxiliatrice Badaróc \& Andressa Bianchi Gumier ${ }^{c}$ \\ ${ }^{a}$ Universidade do Estado do Rio de Janeiro, Rio de Janeiro, Rio de Janeiro, Brasil, \\ ${ }^{b}$ Universidade Salgado de Oliveira, Rio de Janeiro, Rio de Janeiro, Brasil \\ \& ${ }^{c}$ Universidade Federal de Juiz de Fora, Juiz de Fora, Minas Gerais, Brasil
}

\begin{abstract}
Resumo
Vários estudos têm demonstrado que a consciência morfológica está associada à aquisição da leitura e a escrita. Porém, há diferentes tipos de morfemas. O presente estudo explora diferenças no desenvolvimento da consciência morfológica derivacional e flexional no português do Brasil. A amostra do estudo foi constituída por 134 crianças de primeiro ao quarto ano do Ensino fundamental. As crianças realizaram tarefas que acessavam seu conhecimento da morfologia derivacional e flexional. Os resultados mostraram que para todos os anos as tarefas de morfologia flexional foram mais fáceis do que as de derivação. Houve uma melhora no desempenho do segundo para o terceiro ano, mas não do terceiro para o quarto ano. De um modo geral, os resultados corroboram os já encontrados no inglês e no francês.

Palavra-chave: Morfologia derivacional, morfologia flexional, consciência morfológica, consciência metalinguística.
\end{abstract}

\begin{abstract}
Several studies have demonstrated that morphological awareness is related to reading and writing acquisition. However, there are different types of morphemes. The present study explores differences in derivational and flexional awareness development in Brazilian Portuguese. The sample in this study was constituted of 134 children from $1^{\text {st }}$ to $4^{\text {th }}$ grades of primary education who accomplished tasks which assessed their knowledge of derivational and flexional morphology. The results show that for all grades flexional morphology tasks were easier than derivational morphology ones. Performance improved from second to third grade but not from third to fourth grade. In general, results corroborate those already found in English and French languages.

Keywords: Derivational morphology, flexional morphology, morphological awareness, metalinguístic awareness.
\end{abstract}

Morfemas, como definido pelos lingüistas, são as menores unidades lingüísticas que têm significado próprio. Existem duas grandes classes de morfemas: as raízes e os afixos. A raiz pode ser definida como núcleo mínimo de uma construção morfológica. Os afixos podem ser classi-

\footnotetext{
${ }^{*}$ Endereço para correspondência: Instituto de Psicologia, Universidade do Estado do Rio de Janeiro, Campus Francisco Negrão de Lima, Pavilhão João Lyra Filho, São Francisco Xavier, 524, $10^{\circ}$ andar, Bloco B, Sala 10.019, Maracanã, Rio de Janeiro, RJ, Brasil 20550900. E-mail:mmotapsi@gmail.com, silvia_brilhante@yahoo. com.br, carolfconti@yahoo.com.br, tiagonunes.psico@ yahoo.com.br, eonicebrezende@yahoo.com.br, silvinha. amorim@hotmail.com, livinhact@hotmail.com, auxiliatrice@hotmail.com e andressabianchi@hotmail.com
}

ficados em dois tipos: prefixos, afixos adicionados antes da raiz, ou sufixos, afixos adicionados depois da raiz. Assim, a palavra "enraizamento" tem três morfemas "en", "raiz" "mento" e a palavra "raiz" apenas um (Laroca, 2005).

Os morfemas também podem ser classificados como flexões ou derivações. Esses morfemas acoplados a raiz formam novas palavras. As flexões são sufixos que determinam o gênero e o número nos substantivos e adjetivo, e nos verbos constituem os sufixos temáticos, modo-temporais e número-pessoais. As derivações, por sua vez, podem ser: prefixos (ex., "refazer") ou sufixos (ex., "leiteiro"). As flexões têm um caráter morfossintático e possuem uma estabilidade semântica, já as derivações tratam da estrutura das palavras, neste caso pode haver extensões do sentido destas palavras (ver Laroca, 2005, para uma revisão). 
Esta distinção é importante porque nas derivações as palavras muitas vezes mudam de classe gramatical. Por exemplo, a palavra "belo" (que é um adjetivo) pode se tornar "beleza" (que é um substantivo abstrato) pelo processo de derivação. No caso das flexões, as palavras não mudam de classe gramatical. Além disso, há regras gramaticais de formação de palavras flexionadas, o que nem sempre acontece com as derivações. Os verbos no passado sempre terminam com o morfema "am" (e.g., "comeram"), enquanto os verbos no futuro recebem o morfema "ao" (e.g., "comerão") Por fim, um mesmo morfema derivacional pode ter significados ligeiramente diferentes dependendo das palavras em que se encontram. Por exemplo, o "eiro" em "açucareiro" e em "leiteiro" (Deacon \& Braynt, 2005).

É importante saber como os morfemas são processados e como o desenvolvimento desse processamento ocorre. Consciência morfológica é a habilidade de refletir sobre os morfemas que compõem as palavras. Vários estudos mostram que a consciência morfológica está associada à leitura e escrita (Carlisle, 1995, 1996, 2000; Deacon \& Kirby, 2004; Nagy, Berninger, \& Abbot, 2006; Mota, 2012; Mota, Anibal, \& Lima, 2008; Mota, Santos, et al., 2008; Mota \& Silva, 2007; Mota et al., 2012). Levando em conta as características dos diferentes tipos de morfema (flexionais e derivacionais), podemos pensar que o desenvolvimento da capacidade de refletir sobre a morfologia derivacional pode ser diferente do desenvolvimento do processamento da morfologia flexional, e, portanto, pode ser diferente também a influência que este tipo de processamento tem na aquisição da leitura e escrita.

Casalis e Louis-Alexandre (2000) lembram que os principais aspectos da morfologia flexional são adquiridos pela criança antes de começarem a se alfabetizar, enquanto a morfologia derivacional continua a se desenvolver até o final do Ensino Fundamental. Se o desenvolvimento do conhecimento morfológico ocorre de forma diferente para a morfologia derivacional e flexional, é possível que o mesmo ocorra com a consciência morfológica derivacional e flexional. Isto é, a habilidade de refletir sobre os processos de derivação e flexão se desenvolveria de maneiras diferentes. Se o argumento de Casalis e Louis-Alexandre estiver correto, o desenvolvimento da morfologia derivacional deverá acontecer mais tardiamente do que o desenvolvimento da morfologia flexional.

Carlisle (1995) deu a crianças de educação infantil e primeira série uma tarefa de produção morfológica. Nesta tarefa a criança tinha que completar uma frase com uma palavra faltando. À criança era apresentada uma palavra como, por exemplo, "fazenda", e ela tinha que completar uma frase como "Meu tio é um?". Um terço das palavras omitidas eram flexões, um terço eram palavras derivadas com uma relação fonologicamente transparente com a palavra alvo, e o outro terço eram de palavras derivadas, mas com uma relação opaca com a palavra alvo. Os resultados mostraram que para todas as séries era mais fácil completar as frases que requeriam as flexões do que as derivações. As crianças da educação infantil tiveram muitas dificuldades em resolver esta tarefa. O índice de acerto foi de 1,9\% para as palavras derivadas com relações opacas, $22,8 \%$ para as palavras derivadas e $36,5 \%$ para as palavras flexionadas.

Estes resultados indicam que as crianças pequenas têm dificuldades em resolver tarefas que envolvem a produção de palavras morfologicamente complexas. Tarefas de produção envolvem um conhecimento mais explícito da morfologia da língua. Esses resultados indicam também que o processamento das flexões é mais fácil para as crianças do que as derivações.

Outro estudo sobre esse tema foi investigado por Deacon e Bryant (2005). Esses pesquisadores deram as crianças de cinco a oito anos de idade um teste de escrita, no qual as crianças tinham que escrever palavras morfologicamente simples (com um morfema) e palavras morfologicamente complexas (com dois morfemas). Metade das palavras de dois morfemas era de palavras derivadas e a outra metade eram palavras flexionadas. As palavras tinham o mesmo som final. Assim, a palavra notion (com um morfema) e a palavra connection (com dois morfemas) terminavam com o mesmo som. Os autores predisseram que se as crianças processam a morfologia da língua, elas teriam uma facilidade maior em escrever o som final das palavras quando eles eram morfemas, do que quando não eram. Já que o som final das palavras era o mesmo, qualquer diferença nos resultados só poderia ser atribuída ao processamento morfológico da palavra.

Os resultados desse estudo mostraram que as crianças escreviam mais corretamente os sons finais das palavras quando eram morfemas do que quando não eram. Porém, a análise do tipo de morfema escrito mostrou que este resultado era referente à apenas as flexões. Deacon e Bryant (2005) concluíram que a facilitação encontrada na escrita de um dos dois tipos de morfemas possivelmente ocorria porque na morfologia derivacional há uma mudança na classe gramatical das palavras morfologicamente complexas, o que não ocorre com a morfologia flexional. Assim, seria mais fácil para as crianças entender as relações morfêmicas nas flexões do que nas derivações.

Embora, evidências demonstrem que a morfologia flexional se desenvolve mais cedo do que a derivacional, porém, esse desenvolvimento poderia ser diverso para diferentes ortografias, uma vez que há evidências de que características das línguas afetam o desenvolvimento da consciência morfológica. Por exemplo, Duncan, Casalis, e Colé (2009) testaram crianças francesas e inglesas de primeiro ao terceiro ano em diferentes tarefas de consciência da morfologia derivacional. Os resultados mostraram que as crianças francesas tiveram de um modo geral desempenho superior ao das inglesas. Esses resultados são explicados com base nas características do sistema de formação de palavras das duas línguas. Uma questão que pode ser levantada é, dada as características da ortografia do português, como se daria o desenvolvimento da 
consciência morfológica nessa ortografia, em particular, os padrões de desenvolvimento da morfologia flexional e derivacional encontrados no inglês e no francês poderiam ser repetidos no português?

Assim, os estudos que investigam o desenvolvimento da consciência morfológica derivacional e flexional parecem indicar que a morfologia flexional se desenvolve antes da morfologia derivacional. Até onde pudemos revisar nenhum estudo investigou essa questão para o português do Brasil. Esse é o objetivo do presente trabalho.

\section{Método}

\section{Participantes}

Participaram dessa pesquisa uma sub-amostra de 134 crianças do projeto "Avaliação Psicométrica de Medidas de Consciência Metalingüística". As crianças tiveram autorização para participar da pesquisa assinada por seus responsáveis segundo as normas do Comitê de Ética e Pesquisa / protocolo de número 1683.027.2009. Além da concordância dos pais, as crianças também foram elucidadas sobre a participação voluntária na pesquisa. A amostra do estudo acabou constituída por 134 crianças, de ambos os sexo, entre 6 e 13 anos (idade média= 101,82 meses; desvio padrão $=10,48$ meses) do segundo ao quarto ano do ensino fundamental, regularmente matriculadas em três escolas públicas do interior de Minas Gerais. A média de idade das crianças do segundo ano foi de 89,2 meses $(D P=1,38$ meses), do terceiro ano de 99,7 meses ( $D P=0,78$ meses) e do quarto ano 111,23 ( $D P=0,9$ meses).

\section{Instrumentos}

\section{Instrumentos para Avaliar a Consciência \\ Morfossintática}

Analogia gramatical derivacional (adaptado de Nunes, Bindman, \& Bryant, 1997). A tarefa analisa a capacidade da criança em manejar os morfemas. A tarefa foi adaptada, em um estudo não publicado por Mota et al. (2010) considerando a especificidade da morfologia derivacional da língua portuguesa. Nesse sentido, foram selecionados oito itens, para melhorar a consistência interna da tarefa. A análise de consistência interna apresentou índices adequados, mas com carência, com alfa de Cronbach referente a 0,67 . Em cada um, a criança deverá ser capaz de formar uma palavra morfologicamente complexa a partir de uma palavra alvo. Por exemplo, tênis-tenista; arte-? Cabe explicar que as palavras morfologicamente complexas são aquelas compostas por mais de um morfema, por exemplo, florzinha $=$ flor (raiz) + inha (sulfixo). Assim, essa tarefa foi organizada em duas partes, uma abrangendo morfológica derivacional e a outra flexional. Os critérios estabelecidos para correção contam com um ponto para cada acerto e zero ponto para cada erro.

Tarefa de analogia flexional (Nunes et al., 1997). A tarefa adaptada por Mota (1996) requerer da criança a capacidade de identificar a modificação morfossintática num par de palavras e depois aplicá-la em outro par. A relação morfossintática entre as palavras devem ser mantidas pelas crianças. A análise de consistência interna apresentou em um estudo não publicado por Mota et al. (2010) índices adequados, mas com carência, com alfa de Cronbach referente a 0,63 . Essa tarefa foi organizada no total de oito itens, nos quais dois referem à concordância verbal, quatro de flexão de número e dois de gênero. Para cada do item correto foi dado um ponto e para cada erro zero pontos.

\section{Procedimento}

As aplicações das tarefas e testes selecionados para o Projeto original ocorreram em locais reservados nas próprias escolas e foram organizados em quatro momentos: três individuais e um coletivo. As aplicações aconteceram em aproximadamente 50 minutos para cada encontro. A escolha por este número de sessões foi devido à quantidade de tarefas e o possível cansaço das crianças ao realizá-las, o que poderia comprometer a fidedignidade das respostas.

A tarefa de Analogia Gramatical Derivacional foi aplicada individualmente. Para sua aplicação, foram apresentadas à criança um par de palavras relacionadas entre si, depois ela ouviu mais um palavra na qual deveria completar com outra. "Por exemplo, pedra-pedreiro, leite - ?" e assim sucessivamente com os outros pares de palavras.

A Tarefa de Analogia flexional foi aplicada de forma semelhante a de morfologia derivacional. Nesse caso, foram apresentados dois pares de palavras relacionados, e depois foi dito uma palavra à criança para que ela completasse com outra palavra. As palavras a serem produzidas envolviam a flexão da palavra do par. "Por exemplo, homem-mulher / Ele - ?"

\section{Resultados}

A análise das médias apresentada na Tabela 1 mostra um aumento no desempenho nas tarefas de consciência morfológica com os anos do ensino fundamental. Mostra também que as crianças acharam mais fácil produzir a resposta correta para os pares que envolviam as palavras flexionadas do que as derivadas.

Tabela 1

Média e Desvio Padrão para o Número de Respostas Corretas nas Tarefas de Analogia Flexional e Derivacional

\begin{tabular}{cccc}
\hline & & $\begin{array}{c}\text { Analogia } \\
\text { Derivacional }\end{array}$ & $\begin{array}{c}\text { Analogia } \\
\text { Flexional }\end{array}$ \\
\hline $2^{\text {o }}$ ano $(n=30)$ & Média & $4,13(1,77)$ & $5,60(1,57)$ \\
$3^{\text {o }}$ ano $(n=52)$ & Média & $5,00(1,47)$ & $6,42(1,12)$ \\
$4^{\circ}$ Ano $(n=52)$ & Média & $5,35(1,36)$ & $6,51(1,51)$ \\
\hline Total & Média & $4,94(1,56)$ & $6,27(1,43)$ \\
\hline
\end{tabular}


Mota, M. M. P. E., Guimarães, S. B., Conti, C., Linhares, T., Rezende, L. B., Amorin, S., Coelho, L., Badaró, A. \& Gumier, A. B. (2013). Diferenças entre o Desenvolvimento da Morfologia Derivacional e Flexional no Português Brasileiro no Ensino Fundamental.

Para confirmar se as médias diferiram de forma estatisticamente significativa, uma análise de variância fatorial mista (2x3) foi realizada. A variável dependente foi o número de respostas corretas na tarefa de analogia. O fator de medidas repetidas foi o tipo de morfema com dois níveis (derivacional e flexional) e o fator entre sujeitos ano escolar (segundo, terceiro e quarto ano). Houve um resultado estatisticamente significativo para tipo de morfema $\left(F_{(1,131)}=82,3, p<0,001\right)$, as crianças acertaram mais itens para as palavras flexionadas do que para as palavras derivadas. Para o fator ano escolar também houve um resultado estatisticamente significativo $\left(F_{(2,131)}\right.$ $=8,05, p<0,001)$. Análises pós hoc Tukey mostraram que os escores do segundo ano foram inferiores ao do terceiro ano $(p<0,05)$ e os do quarto ano $(p<0,05)$. O terceiro e quarto ano não diferiram entre eles $(p>0,05)$. A interação tipo de morfema $\mathrm{x}$ ano escolar não foi significativa $\left(F_{(2,131)}\right.$ $=0,41, p=0,66$ ).

\section{Discussão}

O objetivo do presente estudo foi investigar o desenvolvimento da habilidade de refletir sobre a morfologia derivacional e flexional no português do Brasil. A hipótese levantada com base nas características dos dois tipos de morfema, flexionais e derivacionais, era a de que o desenvolvimento da capacidade de refletir sobre morfologia derivacional seria diferente da capacidade de refletir sobre a morfologia flexional. A morfologia derivacional demoraria mais tempo a se desenvolver por ser um sistema aberto, sem regras gramaticais claras de formação de palavras para a maioria dos casos, enquanto há regras claras sobre a formação de palavras flexionadas.

Os resultados encontrados mostraram que as crianças de todas as séries escolares acharam mais fácil produzir uma resposta correta quando a tarefa envolvia a morfologia flexional do que quando envolvia a morfologia derivacional. Esses achados são compatíveis com os já encontrados em outras línguas alfabéticas como o francês e o inglês (Casalis \& Louis-Alexandre, 2000; Deacon \& Bryant, 2005). Sugerem que, de fato, as características dos dois sistemas de morfemas podem levar a diferenças no seu processamento e desenvolvimento. É possível que as crianças brasileiras, como as de outras línguas alfabéticas, extraiam e generalizem as regras da morfologia flexional para diferentes palavras, porque suas regras se repetem para várias palavras as tornando salientes. $\mathrm{O}$ mesmo não aconteceria com a morfologia derivacional, pois essas regras variam de palavra para palavra.

Outro ponto observado foi o de que há um desenvolvimento com a escolarização na habilidade de refletir sobre os morfemas. Esse desenvolvimento parece se estabilizar no terceiro ano. Para os dois tipos de morfemas, houve diferenças significativas no desempenho entre o segundo e terceiro e segundo e quarto anos, porém, as médias não diferiram de forma estatisticamente significativa para o terceiro e o quarto ano. Uma explicação possível para esse padrão de resultado pode estar relacionado ao sistema de educacional brasileiro, que intensifica o ensino da gramática por volta do terceiro ano do ensino Fundamental.

O papel do sistema educacional no desenvolvimento da consciência morfológica é um aspecto que precisa ser mais bem investigado. O ensino da gramática ajuda a desenvolver a consciência morfológica ou essa é uma característica que se desenvolve a partir do desenvolvimento da língua escrita? Pode-se pensar que uma vez que o princípio alfabético está consolidado, as crianças comecem a pensar nas razões que fazem com que a ortografia das palavras seja diferente para palavras que tenham sons parecidos (ex. "cobriu" e "fabril"). Muitas dessas razões têm com base a origem das palavras e seu processo de formação, isto é, estão ligados a estrutura morfológica das palavras. Esse processo pode levar as crianças a pensar no processo de formação de palavras. Por outro lado, o ensino da gramática torna explícita a regra de formação de palavras e pode influenciar a performance nas tarefas de consciência morfológica. É preciso testar essas duas hipóteses.

Adicionalmente, o fato de se observar padrões diferentes de desenvolvimento para os dois tipos de morfema podem sugerir que sejam diferentes também a influência que esses tipos de morfema têm na aquisição da leitura e escrita. Estudos realizados no português do Brasil têm demonstrado que os dois tipos de morfema contribuem para leitura e escrita (Mota et al., 2002; Mota, Anibal, et al., 2008). No entanto, a contribuição relativa dos conhecimentos desses dois tipos de sistema morfológico não foi ainda investigada no português. Dessa forma, o presente estudo vem abrir novas perspectivas para estudos futuros nessa área, que vem se constituindo num importante foco de investigação.

\section{Referências}

Carlisle, J. (1995). Morphological awareness and early reading achievement. In L. Feldman (Ed.), Morphological aspects of language processing (pp. 189-211). Hillsdale, NJ: Lawrence Erlbaum.

Carlisle, J. (1996). An exploratory study of morphological errors in children's written stories. Reading and Writing: An Interdisciplinary Journal, 8, 61-72.

Carlisle, J. (2000). Awareness of the structure and meaning of morphologically complex words: Impact on reading. Reading and Writing: An Interdisciplinary Journal, 12, 169-190.

Casalis, S., \& Louis-Alexandre, M. (2000). Morphological analysis, phonological analysis, and learning to read French: A longitudinal study. Reading and Writing: An Interdisciplinary Journal, 12(3), 303-336.

Deacon, S., \& Bryant, P. (2005). What young children do and do not know about the spelling of inflections and derivations. Developmental Science, 8(6), 583-594.

Deacon, S. H., \& Kirby, J. (2004). Morphological awareness: Just 'more phonological'? The roles of morphological and phonological awareness in reading development. Applied Psycholinguistics, 25, 223-238. 
Duncan, L., Casalis, S., \& Colé, P. (2009). Early metalinguistic awareness of derivational morphology: Observations from comparison of English and French. Applied Psycholinguistics, 30, 405-440.

Laroca, M. (2005). Manual de morfologia do português. Campinas, SP: Pontes.

Mota, M. (1996). Children's role of grammatical rules in spelling (Tese de doutorado, Departamento de Psicologia Experimental, Universidade de Oxford, Inglaterra).

Mota, M. (2012). Explorando a relação entre a consciência morfológica, processamento cognitivo e o desempenho na escrita. Estudos de Psicologia (Campinas), 29(1), 97-102.

Mota, M., Andrade, C., Henrique, D., Macedo, S., Atalaia, K., Stephan, F., \& Ferreira, D. (2002). Consciência Sintática e desenvolvimento ortográfico. In Encontros Psicopedagógicos - Anais do IV Seminário de Psicopedagogia da UERJ (Vol. 1, pp. 35-39). Rio de Janeiro, RJ: Universidade do Estado do Rio de Janeiro.

Mota, M., Anibal, L., \& Lima, S. (2008). A Morfologia Derivacional contribui para a leitura e escrita no português? Psicologia: Reflexão e Crítica, 21(2), 311-318.

Mota, M., Santos, A. A., Dias, J., Paiva, N., Mansur-Lisboa, S. F., \& Silva, D. A. (2008). Relação entre consciência morfológica e a escrita em crianças do ensino fundamental. Psicologia em Pesquisa, 2(2), 51-60.

Mota, M., \& Silva, K. (2007). Consciência Morfológica e Desenvolvimento Ortográfico: Um Estudo Exploratório. Psicologia em Pesquisa, 1(2), 86-92.

Mota, M., Toledo, M., Bastos, R., Dias, J., Paiva, N., Mansur-Lisboa, S. F., \& Silva, D. A. (2012). Leitura Contextual e Processamento Metalingüístico no Português do Brasil: Um estudo longitudinal. Psicologia: Reflexão e Crítica, 25(2), 114-120.

Nagy, W., Berninger, V., \& Abbot, R. (2006). Contributions of morphology beyond phonology to literacy outcome of upper elementary and middle-school students. Journal of Educational Psychology, 98(1), 134-147.

Nunes, T., Bindman, M., \& Bryant, P. (1997). Morphological strategies: Developmental stages and processes. Developmental Psychology, 33(4), 637-649. 